\title{
The effect of using awake craniotomy combined with multimodal techniques intraoperative on survival of patients with glioblastoma: a single- center experience
}

\section{Li Yang}

Southern Medical University Nanfang Hospital

Ming Lu

guangdong 999brain hospital

Xinqing Deng

guangdong 999brain hospital

Mingming Yang

guangdong 999brain hospital

hui zhou ( $\square$ 40756036@qq.com )

Guangdong Pharmaceutical University https://orcid.org/0000-0001-5437-7282

\section{Research}

Keywords: awake craniotomy, multimodal technique, glioblastoma, resection, outcomes

Posted Date: February 12th, 2020

DOI: https://doi.org/10.21203/rs.2.23394/v1

License: (c) (1) This work is licensed under a Creative Commons Attribution 4.0 International License.

Read Full License 


\section{Abstract}

Background:We analysed outcomes of cerebral glioblastoma patients undergoing awake craniotomies combined with multimodal techniques for tumour resection, with regards to the extent of resection, functional preservation, and prognosis.

Methods:A retrospective analysis was conducted on adult glioblastoma patients who underwent an awake craniotomy from September 2010 to August 2018 under anaesthesia combined with multimodal techniques.

Results:In total, 81 glioblastoma patient charts were analysed. The most common lesion sites were the frontal lobe $(n=36)$, temporal lobe $(n=17)$, and parietal lobe $(n=6)$. The main symptoms were headache $(n=51)$, dyskinesia $(n=11)$, speech disorder $(n=9)$, and epilepsy $(n=10)$. The extent of resection was gross total for $91.36 \%$ patients, subtotal for $7.41 \%$, and partial for $1.23 \%$. No deaths occurred 30 days postoperation. Intracranial haemorrhage occurred in 2 patients, seizures in 5 patients, and intracranial infections in 3 patients. There was no significant difference between preoperative and postoperative Karnofsky Performance Status scores $(P>0.05)$. There were no significant changes in postoperative neurological function in 50 patients. Symptoms improved in 24 patients. Three patients exhibited motor dysfunction, 2 exhibited speech deficits, and 2 exhibited sensory deficits. The average duration of hospitalization was $6.89 \pm 2.66$ days. The shortest survival time was 4 months, the longest survival time was 26 months, and the median survival time was 12 months.

Conclusions: Awake craniotomy using multimodal techniques such as neuronavigation, intraoperative ultrasound, electrophysiology, and tumour fluorescence during an operation can maximize safety during the cerebral glioblastoma resection, thus protecting brain function and improving surgical efficacy and patients' postoperative quality of life.

\section{Background}

Glioblastomas are the most common type of primary malignant brain tumour, with a poor prognosis. ${ }^{1}$ Surgical excision of the tumour is critical to ensure satisfactory results, and "the extent of safe maximum resection" principle has a significant impact on the overall and progression-free survival. $2,3,4,5$

Unfortunately, in many cases, it is difficult to maximize tumour resection while protecting brain function. ${ }^{6}$ Although traditional surgical methods can identify cortical lesions based on sulci, gyri, or cortical vessels, lesions in the subcortical white matter lack structures. ${ }^{7}$ Therefore, it is challenging to achieve safe maximum resection by separating glioblastomas from the surrounding brain using only the naked eye or microscopes.

To overcome this problem, the neuronavigation system was developed. However, the navigation system relies on preoperative images, and intraoperative brain displacement may occur after dural opening and tumour resection, leading to inaccurate positioning. ${ }^{8}$ Intraoperative registration of the navigation system 
can correct these changes when intraoperative magnetic resonance imaging (iMRI) is available. Nevertheless, iMRI cannot provide real-time data on the resection process, and its high costs prevent its widespread use in most neurosurgical theatres. ${ }^{9}$ Therefore, inaccuracy of navigation also affects intraoperative localization of tumour boundaries.

Intraoperative ultrasound (iUS) has been used as an imaging technique in neurosurgery for decades. It is cheap and provides real-time intraoperative information. ${ }^{10}$ However, the resolution of iUS images is poor and it has a limited intraoperative field of vision. ${ }^{11}$ Further, determining the anatomical direction of residual tumours can be challenging.

Fluorescently guided excision of 5-aminolevulinic acid (5-ALA) was another breakthrough for glioblastoma surgery. 5-ALA induces the synthesis and accumulation of porphyrins in glioblastoma tissues, allowing surgeons to observe tumour boundaries under a blue light (wavelength $375-400 \mathrm{~nm}$ ) emission microscope. Tumour resection increases in the observed fluorescent regions. However, 5-ALA is not a reliable marker for low-grade glioblastomas. ${ }^{12}$

In recent years, awake craniotomy has been performed to minimize the possibility of long-term neurological dysfunction. ${ }^{8-10}$ This method reduces intensive care time, total length of hospital stay, and medical costs relative to those of standard methods under general anaesthesia. A more important advantage of this method is that it can be used to protect the functional area by directly stimulating the brain tissue for conscious questioning during the operation. This method of real-time evaluation of intraoperative neurological function can improve postoperative outcomes, specifically, reduce the residual tumour volume and postoperative neurological dysfunction. ${ }^{13,14,15}$

Due to the lack of clear evidence to support the superiority of any single method and the shortcomings of the aforementioned methods, we present a multimodal technique for awake craniotomy, which combines neuronavigation, neurophysiologic monitoring, iUS, and tumour fluorescence. This approach could maximize the safety of tumour resection, protect neuronal function, and improve patients' quality of life and survival. The aim of this study was to retrospectively analyse outcomes in cerebral glioblastoma patients undergoing awake craniotomies combined with multimodal techniques.

\section{Methods}

\section{Patients}

After receiving approval from the institutional review committee, we retrospectively analysed the clinical data of 81 patients who underwent intracranial glioblastoma resection under conscious craniotomy with multimodal techniques from September 2010 to August 2018. All patients were over 18 years of age, were informed of the study's objectives, and provided informed consent. 
All patients included had no surgical contraindications. Preoperative motor function and speech of the patients were normal to ensure they could be fully cooperative. Preoperative diagnosis was based on their performance, medical history, and preoperative MRI, whereby glioblastoma was highly suspected. Postoperative pathology reported World Health Organization (WHO) grade IV glioblastoma. Pathology of the surgical sample was determined by a pathologist according to WHO criteria.

The tumours were located in the supratentorial region, adjacent to the eloquent structures, which included pre- and post-central gyrus, centrum semiovale, corona radiata, internal capsule, basal ganglia, thalamus, Wernicke's area, and Broca's area. The surgeon and anaesthesiologist preoperatively assessed whether the patients were able to cooperate during the operation, by ensuring their KPS (Karnofsky Performance Status) scores were $>70$. All patients were willing to undergo awake craniotomy, and were followed up on time after surgery.

\section{Preoperative preparation}

The KPS score was assessed in all patients, and they underwent an MRI (1.51T or 3.0T GE, USA) before surgery. In patients with tumours near or involving the motor, sensory, or eloquent areas, diffusion tensor imaging (DTI) and blood oxygenation level dependent (BOLD) functional MRI (fMRI) was obtained to delineate fibre tracking of the motor pathways as well as the associated speech pathways.

MRI datasets were transferred to the neural navigation system (Brainlab, AG, Munich, Germany) preoperation. Three-dimensional reconstruction details of the brain were created, which combined T1weighted (T1W) and T2-weighted (T2W) images, and DTI after angiography to delineate the relevant eloquent subcortical structures and three-dimensional boundaries of the tumour.

\section{Surgical technique}

On the morning of the surgery, the anaesthesiologist met the patient. Once the patient was determined to be a suitable candidate for the awake anaesthesia technique, they were prepared for scalp block and gentle sedation (monitoring anaesthesia care [MAC]). When the patient was in the operating room, the anaesthesiologist typically used a cranial scalp block with procaine and adrenaline. Patients were positioned either in the supine or lateral prone position depending on the location of the tumour, and the head was fixed in the Mayfield system with a three-pin head clamp.

The patient was put under general anaesthesia using a laryngeal mask. Bispectrallndex (Bispectrallndex ${ }^{\mathrm{TM}}$, USA) was used to monitor consciousness. A craniotomy flap was drawn after the neuronavigation registration process was completed. The scalp was prepared using the standard aseptic technique. The surgical sites were prepped and covered aseptically. Coverage of the surgical field was performed technically to expose the face to ensure airway patency, facilitate intraoperative motor/speech testing, and minimize rebreathing of carbon dioxide. After the flap was opened, the dura was suspended, the laryngeal mask was removed, and the patient was awakened. Dexmedetomidine and remifentanil were used as sedatives. 
The dura was anesthetized locally with procaine and pierced with a small knife. As planned preoperatively, the catheters were guided by navigation and iUS into the brain to locate the boundary of the tumour. The entry points of the catheters on the cerebral cortex were kept away from the eloquent cortical areas (language/motor/sensory cortex), sulcus, and cortical vessels.

Then, stimulation was performed using an Ojemann stimulator (Radionics, Mass.) with 5-mm spaced bipolar tips. The stimulation parameters were constant current square wave, biphasic pulses of $500 \mathrm{~ms}$ duration at a frequency of $50 \mathrm{~Hz}$. For cortical stimulation, outputs started at $2 \mathrm{~mA}$ and increased by $2 \mathrm{~mA}$ until either the clinical response was observed, $10 \mathrm{~mA}$ output was reached, or clinical seizures occurred.

After avoiding the cortical mapping of the functional area, the fluorescent yellow tumour tissue was removed under the microscope to protect the necessary blood vessels. When no yellow-stained tissue was excised, brain function was again monitored by subcortical stimulation and continuous physical examination. For subcortical stimulation, the $1 \mathrm{~mm}-1 \mathrm{~mA}$ rule was used to estimate the relevant distance between the fibres and stimulus probe. When the distance was less than $1 \mathrm{~cm}$, subcortical stimulation was repeated every $2-3 \mathrm{~mm}$.

If subcortical stimulation reached the 4-mA threshold and/or motor/speech responses or physical examination revealed signs of decreased nerve function, the excision was stopped. If the subcortical stimulation threshold was redetermined or the decrease in nerve function did not improve within 5 minutes, excision was completely terminated. If no other indication was given, excision was performed until the boundary marked by the catheter was reached. All surgeons considered total tumour resection as the surgical target, and the final degree of resection was determined according to intraoperative neuronavigation, DTI, fMRI, iUS, fluorescent visualization of tumour tissues under the surgical microscope, and the degree of damage of language/motor function in patients. After the operation, the bleeding was allowed to stop. The patient was subsequently sedated during craniotomy closure.

\section{Statistical analysis}

Information on patients' age at surgery, sex, tumour histology, procedure type, location, surgery duration, intraoperative events, postoperative course, duration of hospital stay, extent of resection, and postoperative complications were recorded. Tumour location was determined based on MRI images obtained for each patient. The extent of tumour infiltration was recorded according to hemisphere and lobe involvement. The prognosis was determined by the surgical resection area, length of hospital stay, postoperative KPS scores, postoperative mortality, and complications (occurred within 30 days after the surgery, including wound infections, haemorrhage, neurological deficits, and seizures).

MRI re-examination was performed within 48 hours after surgery to assess the extent of tumour resection. It was divided into gross total, near-gross total, or partial by Sanai and Berger.16 If there was no residual signal abnormality, it was considered as a tumour resection. If less than $10 \mathrm{ml}$ of the residual tumour was present, it was considered as a near total resection. If more than $10 \mathrm{ml}$ of residual tumour 
was present, it was considered as a partial. A follow-up MRI was also obtained at approximately 1 month for radiation planning (if applicable). All patients were followed up on for more than 1 year.

Neurological deficits after the surgery were determined according to preoperative and postoperative KPS scores (7 days after surgery), and were designated as improved, unchanged, or worsened. For any patient with a worsened neurological status, if the neurological deficits were relieved within 3 months after the operation, the neurological dysfunction was considered temporary; otherwise, it was deemed persistent.

Descriptive statistics are provided as median, mean, and standard deviations for continuous variables, and frequency of distribution for categorical variables. A Pearson's chi-square test was used for categorical analyses, and a factorial one-way ANOVA test was used for comparing continuous variables. All statistics were performed using SPSS 20.0 (IBM, USA). A value of $\mathrm{P}<0.05$ was considered statistically significant.

\section{Results}

From September 2010 to August 2018, a total of 81 glioblastoma patients who received their first sober craniotomy were analysed. There were 51 males and 30 females, ranging between 18 to 71 years of age with an approximately Gaussian distribution. Median age was $46.15 \pm 12.10$ years. In total, 75 patients were right-handed, and six were left-handed. Tumours were located on the right side of the brain in 47 patients and on the left side of the brain in 34 patients. The most common lesion sites were the frontal lobe $(n=36)$, temporal lobe $(n=17)$, parietal lobe $(n=6)$, occipital lobe $(n=7)$, frontotemporal lobe $(n=7)$, frontoparietal lobe $(n=4)$, and temporoparietal lobe $(n=4)$. The main symptoms were headache $(n=51)$, dyskinesia $(n=11)$, speech disorder $(n=9)$, and epilepsy $(n=10)$. Preoperative KPS scores were 70 in 3 cases, 80 in 6 cases, 90 in 43 cases, and 100 in 29 cases (Table 1).

All patients successfully completed the awake craniotomy (Fig. 1). During the operation, the patients' speech/movement was fully coordinated, and only 2 patients had intraoperative seizures. According to navigation guidance, the motor area in 5 patients, sensory area in 3 patients, and speech area in 2 patients were successfully located by neuro-electrophysiological monitoring during surgery, which were consistent with preoperative DTI and BOLD (Fig. 2). The duration of operation, from the time the from scalp incision was made to the end of operation, was less than 3 hours in 48 patients (59.26\%) , 3-5 hours in 29 patients $(35.80 \%$ ), and more than 5 hours in 4 patients $(4.94 \%)$. The average operation time was $3.02 \pm 1.29$ hours. Postoperative resection ranges were $74(91.36 \%)$ for total resection, six $(7.41 \%)$ for subtotal resection and one (1.23\%) for partial resection (Table. 2). All postoperative pathological reports showed WHO grade IV glioma.

All patients were awake when they left the operating room. No deaths occurred 30 days after operation. Intracranial haemorrhage occurred in 2 patients, seizures in 5 patients ( 3 patients had epileptic symptoms before the operation), and intracranial infections in 3 patients (cured after antibiotic treatment). There were no significant postoperative changes in neurological function in 50 patients, and symptoms improved in 24 patients (Fig. 3). KPS scores on the 7th day after operation were 70 in 2 cases, 80 in 5 
cases, 90 in 33 cases, and 100 in 41 cases. There was no significant difference between preoperative and postoperative KPS scores ( $P>0.05$ ) (Table 3). Three patients had motor dysfunction, 2 had speech deficits, and 2 had sensory deficits. Six patients recovered neurological function within 3 months after operation. One patient had permanent motor dysfunction and no permanent speech deficits. The average duration of hospitalization was $6.89 \pm 2.66$ days, 62 patients were discharged within 7 days, 12 patients were discharged within 7-10 days, and only 7 patients were discharged after more than 10 days (Table 2). The average follow-up time was $14.85 \pm 5.30$ months. The shortest survival time was 4 months. The longest survival time was 26 months. The median survival time was 12 months (Fig. 4).

\section{Discussion}

Gliomas are one of the most common type of intracranial malignant tumours. Although the incidence of gliomas is relatively low (approximately 661/100,000), disability and mortality rates associated with it are very high. ${ }^{17}$ As a high-grade glioma, glioblastomas are characterized by highly invasive growth, difficulty to perform a complete surgical resection, high recurrence rate, and poor prognosis. ${ }^{18}$ Therefore, methods to achieve a breakthrough in the treatment of glioblastoma and improve the prognosis of glioblastoma patients have become an area of intense clinical research.

At present, the main treatment strategy for glioblastoma is to remove as much of the lesion as possible and provide comprehensive treatment such as chemo-radiotherapy to prevent neurological dysfunction. Surgical resection usually includes total, subtotal, or partial resection. Therefore, maximal safe resection of tumours has become the consensus for surgical treatment of glioblastoma. ${ }^{19,20}$ Although the effects of glioblastoma prognosis vary, the amount of residual postoperative tumour is an important factor affecting prognosis. Total resection of tumours directly affects the recurrence and survival time, and the extent of resection may determine subsequent treatment. ${ }^{21}$ Retaining sensation, speech, movement, and other important neurological functions while maximizing tumour removal is a goal of many neurosurgeons.

New technologies are being used to increase the safety and effectiveness of surgery. In particular, performing an awake craniotomy using neuronavigation, cortical electrical stimulation, intraoperative ultrasound, tumour luciferin, and other auxiliary means, has greatly expanded the neurosurgeon's expertise in tumour surgery of eloquent eloquentregions, which promotes tumour removal as thoroughly as possible. ${ }^{22}$ Initially, when the neuronavigation system became standard, it allowed surgeons to preoperatively create a "desired range" of excision, whether total, subtotal, or partial. However, due to the complication of intraoperative brain displacement, neurophysiological monitoring was added to improve the safety of the operation. ${ }^{23}$

We first identified tumour boundaries with navigation-guided catheterization, assisted neurophysiological monitoring as projection guidance to determine neural function, and "fine-tuned" tumour resection. 5-ALA only provided a relative boundary for glioblastoma, and the surgeon decided to further remove the tumour tissue or to terminate resection based on neurophysiological monitoring results, as well as the patient's 
condition, family support, patient's occupation, and prior discussion with the patient. Since iUS and iMRI cannot provide the "expected excision range", they can only be used as post-excision verification tools.

This study explored the application of awake craniotomy combined with neuronavigation, neuroelectrophysiological monitoring, iUS, and tumour fluorescence for glioblastoma resection to achieve tumour resection with maximum safety. Awake craniotomy had been performed as a routine strategy in our centre, and more than 300 cases had been performed to achieve maximum safety removal while minimizing the rate of neurological damage. ${ }^{24}$ In our initial experience, 80 patients had total or near total resection, which represents a good resection range, comparable to or better than the $91 \%$ of total or near total resection reported thus far. ${ }^{25}$ The rate of new neurological damage has been reported to be $18-23 \%$ has been reported. $26,27,28$ In our study, $91 \%$ of patients had either improved or no change in their neurological status. Only 7 patients experienced new neurological dysfunction and a reduced KPS score after surgery, including worsening of their pre-existing neurological dysfunction. This decline was most likely due to mechanical manipulation and heat damage to the functional brain tissue. However, intraoperative neuroelectrophysiological monitoring and DTI imaging localization were used to protect the eloquent structures of brain tissue, and 6 patients recovered within 3 months after surgery, avoiding permanent neurological damage.

In our patient cohort, all operations were successfully completed, and conversion to general anaesthesia was not required during the operation. The rate of conversion to general anaesthesia or failure has been reported to range from 2.4 to $6.4 \% .{ }^{29}$ In addition, during the study period, awake craniotomy patients were hospitalized for an average of 6.89 days and had fewer postoperative complications. Intraoperative epilepsy is one of the most common complications of awake craniotomy. In our study, 2 patients experienced brief seizures during motor localization, and we quickly controlled the symptoms with cold saline and propofol and continued the surgery. It is currently believed that seizures may be caused by cortical stimulation and localization. Patients with a long history of seizures undoubtedly have a higher probability of intraoperative seizure. Thus, we used cortical stimulation very carefully for intraoperative localization to reduce the risk of intraoperative seizures. We optimized the control of epileptic seizures with the preoperative and intraoperative use of sodium valproate to prevent epilepsy.

Here, a retrospective analysis was conducted on patients undergoing an awake craniotomy. Although the number of cases was limited, our results suggest the promising prospects for performing awake craniotomy to remove glioblastomas. These results at least partly confirmed the effectiveness of awake surgery combined with multimodal techniques in the protection of intraoperative neurological function. Among the 81 glioblastoma patients studied, the median survival time was 12 months, which was higher than the median survival time of glioblastoma patients studied at home and abroad. This partly confirmed the improvement in prognosis and survival rate of glioblastoma patients after total resection and expanded resection. We found that awake craniotomy improved surgical safety and reduced neurological deficits, consistent with past reports. The next step is to study this technique in other patients (e.g., thalamus, basal ganglia, internal capsule, etc.) and establish a control group for comparison in order to demonstrate the direct impact of the technique on the extent of resection, 
postoperative nerve status, or overall survival. In addition, patients' coordination and tolerance during awake craniotomy were enhanced by ensuring comfort during surgery, appropriate use of sedatives to minimize fatigue, and the use of long-duration anaesthetics to block local scalp surgery. Therefore, we were able to achieve the goal of maximum safe resection in the vast majority of patients.

\section{Conclusions}

In conclusion, awake craniotomy combined with neuronavigation, neuroelectrophysiological monitoring, iUS, tumour fluorescence, and other multimodal techniques can effectively protect neurological function and maximise the removal rate of intracranial glioblastomas, thereby providing a new surgical strategy for neurosurgeons.

\section{Declarations}

\section{Availability of data and materials}

All the data used to support the findings of this study are currently under embargo. Requests for data, 6 months after publication of this article, will be considered by the corresponding author. The e-mail address: 40756036@qq.com (Hui Zhou).

\section{Acknowledgements}

The authors thank the entire staff of the Department of neurosurgery, Guang dong 999 brain Hospital,and also thank Editage (www.editage.cn) for English language editing.The corresponding author would like to express special love to his wife, Li Yang M.D.

Author' contributions

HZ: Project development, Data collection and Manuscript writing. LY: Data analysis and Manuscript editing. ML, XQD, MMY: The surgery and anesthesia performed.All authors have read and approved this manuscript, and ensure that this is the case.

\section{Funding}

This study was supported by the Medical Scientific Research Foundation of Guangdong Province, China (B2015106, A2019235). The funding body has no role in the design of the study and collection, analysis, and interpretation of data and in writing the manuscript.

\section{Author information}

Li Yang, Ming Lu and Xinqing Deng contributed equally to this work.

Affiliations 
Department of Nephrology, Nanfang Hospital, Southern Medical University, Guangzhou, Guangdong, China

Li Yang

Department of neurosurgery, Guang dong 999 brain Hospital, Guangzhou, Guangdong, China.

Ming Lu, Xinqing Deng and Mingming Yang

Department of neurosurgery, The First Affiliated Hospital of Guangdong Pharmaceutical University, Guangzhou, Guangdong, China.

Hui Zhou

Corresponding author

Correspondence to Hui Zhou.

\section{Ethics approval and consent to participate}

The study was approved by the ethics committees of Guang dong 999 brain Hospital. Informed consent was obtained from all individual participants included in the study. The informed consent obtained was written.Our research complies with the Declaration of Helsinki.

\section{Consent for publication}

Not applicable.

\section{Competing Interests}

The authors have no conflict of interest in this paper.

\section{Abbreviations}

MRI: Magnetic resonance imaging

WHO: World Health Organization

iUS: Intraoperative ultrasound

GBM: Glioblastoma

5-ALA: 5-aminolevulinic acid

DTI: Diffusion tensor imaging

BOLD: Blood oxygenation level dependent 
fMRI: Functional MRI

BIS: Bispectrallndex

\section{References}

1. Matarredona ER, Pastor AM. Neural Stem Cells of the Subventricular Zone as the Origin of Human Glioblastoma Stem Cells. Therapeutic Implications. J Front Oncol. 2019,9(7):779.

2. Claus EB, Horlacher A, Hsu L, Schwartz RB, Dello-lacono D, Talos F, et al.Survival rates in patients with low-grade glioma after intraoperative magnetic resonance image guidance. J Cancer. 2005,103(6):1227-33.

3. Lacroix M, Abi-Said D, Fourney DR, Gokaslan ZL, Shi W, DeMonte F, et al. A multivariate analysis of 416 patients with glioblastoma multiforme: prognosis, extent of resection, and survival. $\mathrm{J} \mathrm{J}$ Neurosurg. 2001,95(2):190-8.

4. McGirt MJ, Chaichana KL, Gathinji M, Attenello, FJ, Than K, Olivi A, et al. Independent association of extent of resection with survival in patients with malignant brainastrocytoma. J Neurosurg. 2009,110:156-62.

5. Oppenlander ME, Wolf AB, Snyder LA, Bina R, Wilson JR, Coons SW, et al.An extent of resection threshold for recurrent glioblastoma and its risk for neurological morbidity. J J Neurosurg. 2014,120(4):846-53.

6. Hardesty DA, Sanai N. The value of glioma extent of resection in the modern neurosurgical era. J Front Neurol. 2012, 3(3):140.

7. Grabowski MM, Recinos PF, Nowacki AS, Schroeder JL, Angelov L, Barnett G H, et al.Residual tumor volume versus extent of resection: predictors of survival after surgery for glioblastoma. $\mathrm{J} \mathrm{J}$ Neurosurg. 2014,121(5):1115-23.

8. Romano A, D'Andrea G, Calabria LF, Coppola V, Espagnet CR, Pierallini A, et al. Pre- and intraoperative tractographic evaluation of corticospinal tract shift. J Neurosurgery. 2011,69(3):696-704. et al.

9. Li P, Qian R, Niu C, Fu X. Impact of intraoperative MRI-guided resection on resection and survival in patient with gliomas: a meta-analysis. J Curr Med Res Opin. 2017,33(4):621-630.

10. Prada F, Bene MD, Fornaro R, Vetrano IG, Martegani A, Aiani L, et al. Identification of residual tumor with intraoperative contrast-enhanced ultrasound during glioblastoma resection. J Neurosurg Focus.:E7.

11. Trevisi G, Barbone P, Treglia G, Mattoli MV, Mangiola A. Reliability of intraoperative ultrasound in detecting tumor residual after brain diffuse glioma surgery: a systematic review and meta-analysis. $J$ Neurosurg Rev. 2019, 8(14).

12. Cho SS, Salinas R, Lee JYK. Indocyanine-Green for Fluorescence-Guided Surgery of Brain Tumors: Evidence, Techniques, and Practical Experience. J Front Surg. 2019,6(11). 
13. Lu VM, Phan K, Rovin RA. Comparison of operative outcomes of eloquent glioma resection performed under awake versus general anesthesia: A systematic review and meta-analysis. J Clin Neurol Neurosurg. 2018,169(6):121-127.

14. Alimohamadi M, Shirani M, Shariat Moharari R, Pour-Rashidi A, Ketabchi M, Khajavi M, et al.Application of Awake Craniotomy and Intraoperative Brain Mapping for Surgical Resection of Insular Gliomas of the Dominant Hemisphere. J World Neurosurg. 2016,92(8):151-158.

15. Sanai N, Berger MS. Operative techniques for gliomas and the value of extent of resection. $J$ Neurotherapeutics. 2009,6(3):478-86.

16. Kreth FW, Warnke PC, Ostertag CB, Keles GE. The effect of extent of resection on recurrence in patients with low grade cerebral hemisphere gliomas. J Cancer. 1995, 75(11): 2785-7.

17. Schwartzbaum JA, Fisher JL, Aldape KD, Wrensch, M. Epidemiology and molecular pathology of glioma. J Nat Clin Pract Neurol. 2006, 2(9):494-503.

18. Almeida JP, Chaichana KL, Rincon-Torroella J, Quinones-Hinojosa A.The value of extent of resection of glioblastomas: clinical evidence and current approach. J Curr Neurol Neurosci Rep. 2015,15(2):517.

19. Lam S, Lin Y, Zinn P, Su J, Pan IW. Patient and treatment factors associated with survival among pediatric glioblastoma patients: A Surveillance, Epidemiology, and End Results study. J J Clin Neurosci. 2018,47(1):285-293.

20. Mcgirt MJ, Chaichana KL, Gathinji M, Attenello FJ, Than K, Olivi A, et al. Independent association of extent of resection with survival in patients with malignant brain astrocytoma. J J Neurosurg. 2009, 110(1):156-162.

21. Chen LF, Yang Y, Ma XD, Yu, XG, Gui, QP, Xu, BN, et al. Optimizing the Extent of Resection and Minimizing the Morbidity in Insular High-Grade Glioma Surgery by High-Field Intraoperative MRI Guidance. J Turk Neurosurg. 2017,27(5):696-706.

22. Barbagallo, G; Maione, M; Peschillo, S; et al. Intraoperative Computed Tomography, navigated ultrasound, 5-Amino-Levulinic Acid fluorescence and neuromonitoring in brain tumor surgery: overtreatment or useful tool combination? J J Neurosurg Sci. 2019,11(1).

23. Pallud J, Rigaux-Viode O, Corns R, Signorelli F, Visocchi M, Sortino G,et al. Direct electrical bipolar electrostimulation for functional cortical and subcortical cerebral mapping in awake craniotomy. Practical considerations. J Neurochirurgie. 2017, 63(3):164-174

24. Lu M, zhou H, Deng XH, Lu JK, He XJ, Lin DL, et al. Clinical observation of intracranial lesions resected under intraoperative arousal anesthesia (report of 200 cases). J Chinese journal of neurosurg. 2017, 33(03):275-279.

25. Groshev A, Padalia D, Patel S, Garcia-Getting R, Sahebjam S, Forsyth PA, et al. Clinical outcomes from maximum-safe resection of primary and metastatic brain tumors using awake craniotomy. $J$ Clin Neurol Neurosurg. 2017,157(1):25-30.

26. Groshev A, Padalia D, Patel S, Garcia-Getting R, Sahebjam S, Forsyth PA, et al. Clinical outcomes from maximum-safe resection of primary and metastatic brain tumors using awake craniotomy. $J$ 
Clin Neurol Neurosurg. 2017,157(1):25-30.

27. Trinh VT, Fahim DK, Shah K, Tummala S, McCutcheon IE, Sawaya R, et al. Subcortical injury is an independent predictor of worsening neurological deficits following awake craniotomy procedures. $J$ Neurosurg. 2013,72(2):160-9.

28. Gravesteijn BY, Keizer ME, Vincent AJPE, Schouten JW, Stolker RJ, Klimek M. Awake craniotomy versus craniotomy under general anesthesia for the surgical treatment of insular glioma: choices and outcomes. J Neurol Res. 2018,40(2):87-96.

29. Nossek E, Matot I, Shahar T, Barzilai O, Rapoport Y, Gonen T, et al.Failed awake craniotomy: a retrospective analysis in 424 patients undergoing craniotomy for brain tumor. J J Neurosurg. 2013,118(2):243-9.

\section{Tables}

Due to technical limitations, tables 1-3 are only available as downloads in the supplementary files.

\section{Figures}

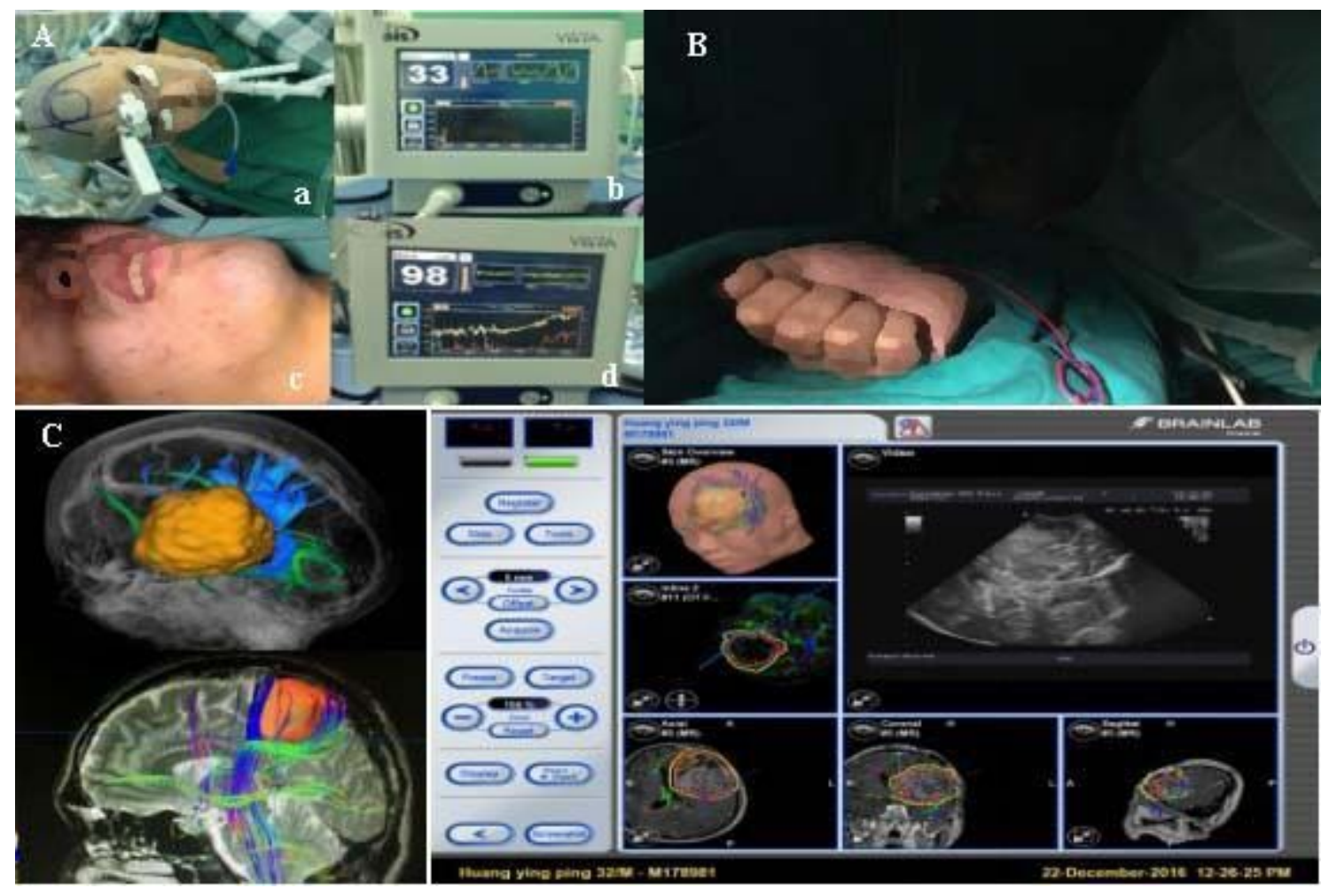

Figure 1

Intraoperative status of patients and the application of electrophysiology and neuronavigation. a. Patient anaesthesia status and bispectral index value (a,b). b. Intraoperative electrophysiology was used to monitor median nerve evoked potential (white arrow). c. Navigation and ultrasound were combined with 
magnetic resonance imaging/diffusion tensor imaging data to develop a tumour localization program before and during surgery

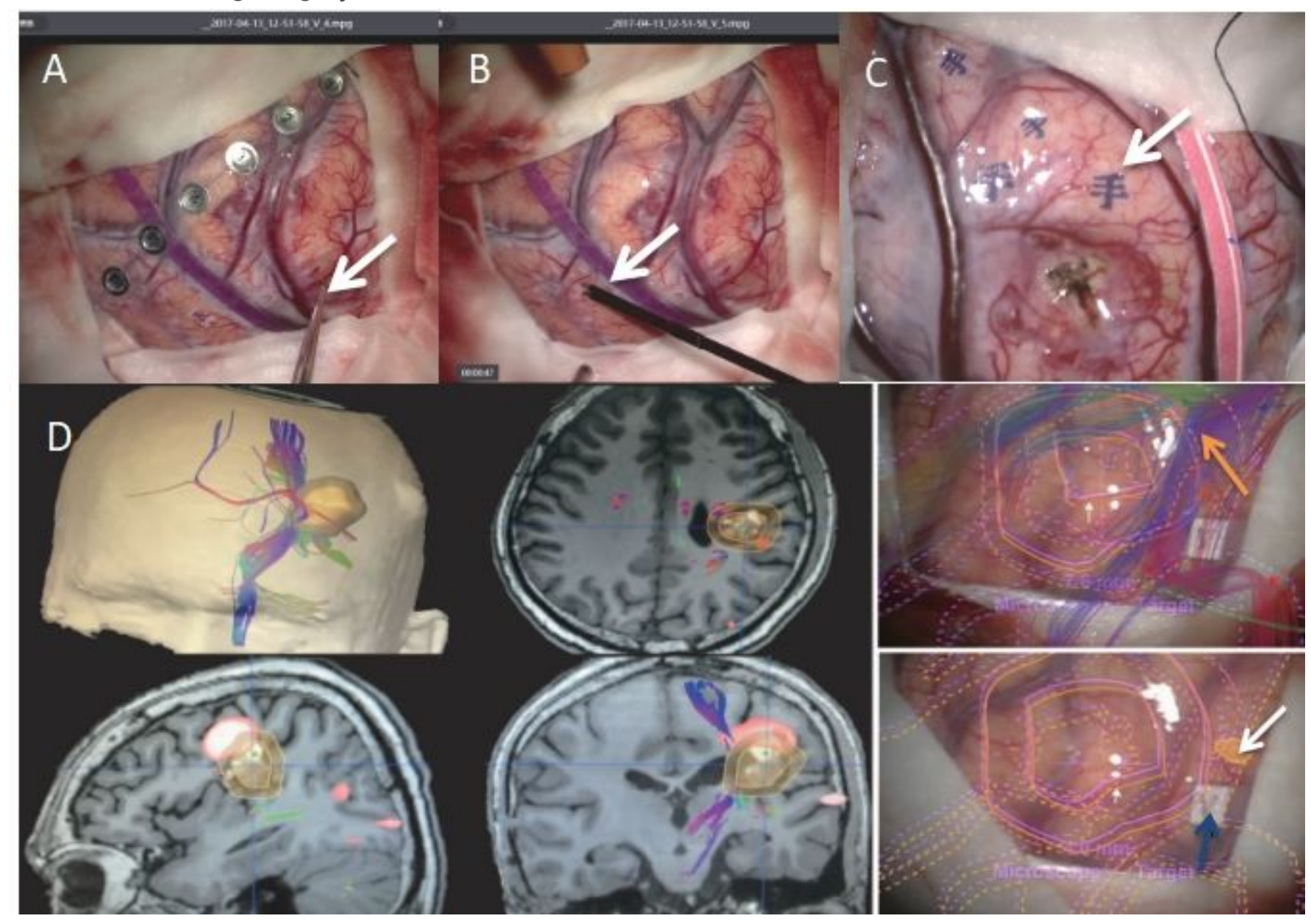

Figure 2

The eloquent areas of the patient's brain located during surgery. a. The navigation bar locates the lesion (arrow). b. Cortical electrical stimulation localizes functional areas of the brain (arrow). c. Upper limb motor area (arrow). d. Intraoperative navigation combined with diffusion tensor imaging and functional magnetic resonance imaging (fMRI) are used to locate the cortical-spinal cord tract (yellow arrow), language area shown by fMRI (white arrow), and actual speech area (blue arrow) 


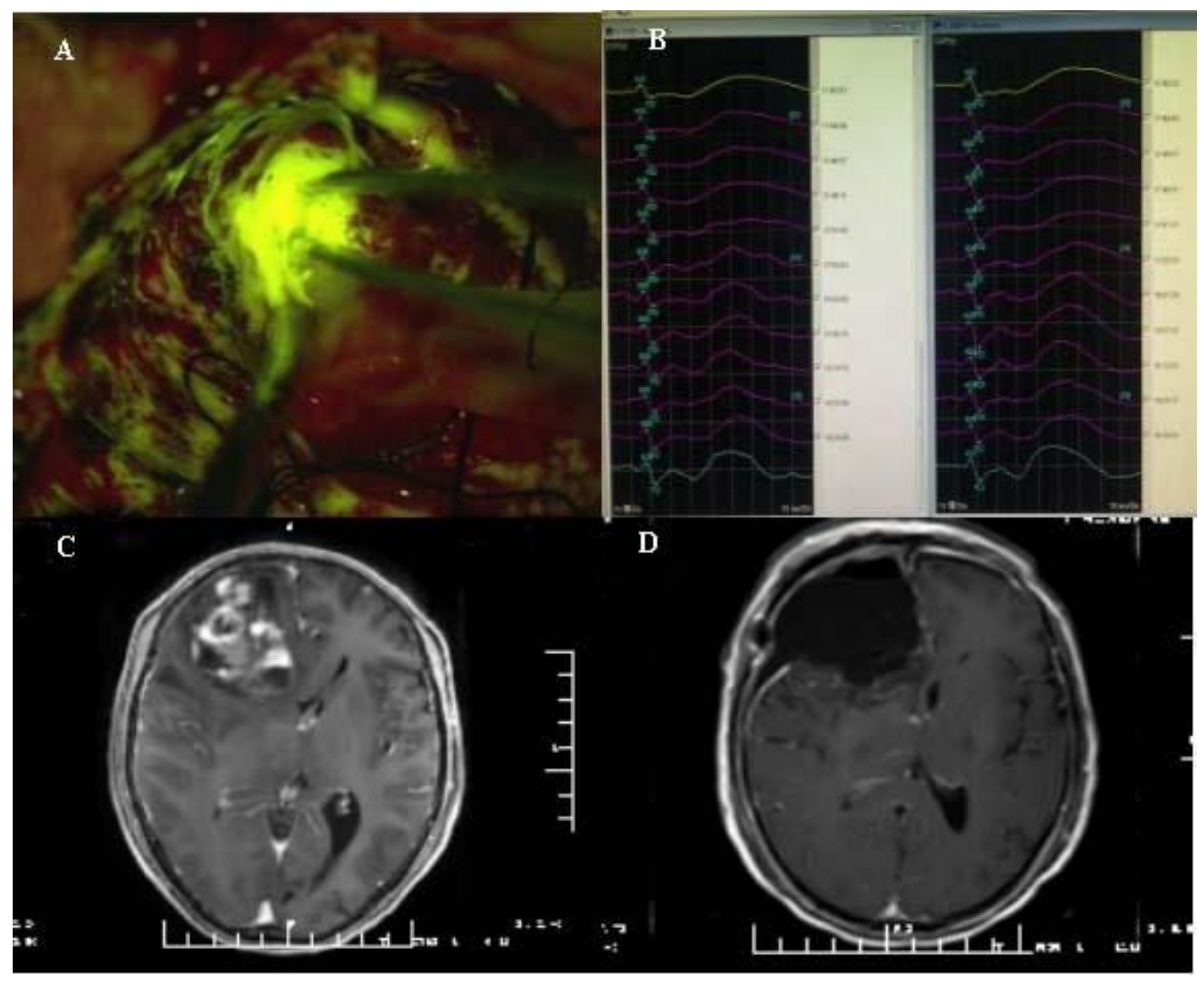

\section{Figure 3}

Extent of tumour resection and preservation of nerve function. a. Intraoperative fluorescence imaging was used to determine tumour boundaries. $b$. There was no change in the amplitude of somatosensoryevoked potentials of the median nerve of the upper limb during surgery. c, d. Postoperative MRI revealed total removal of the tumour 


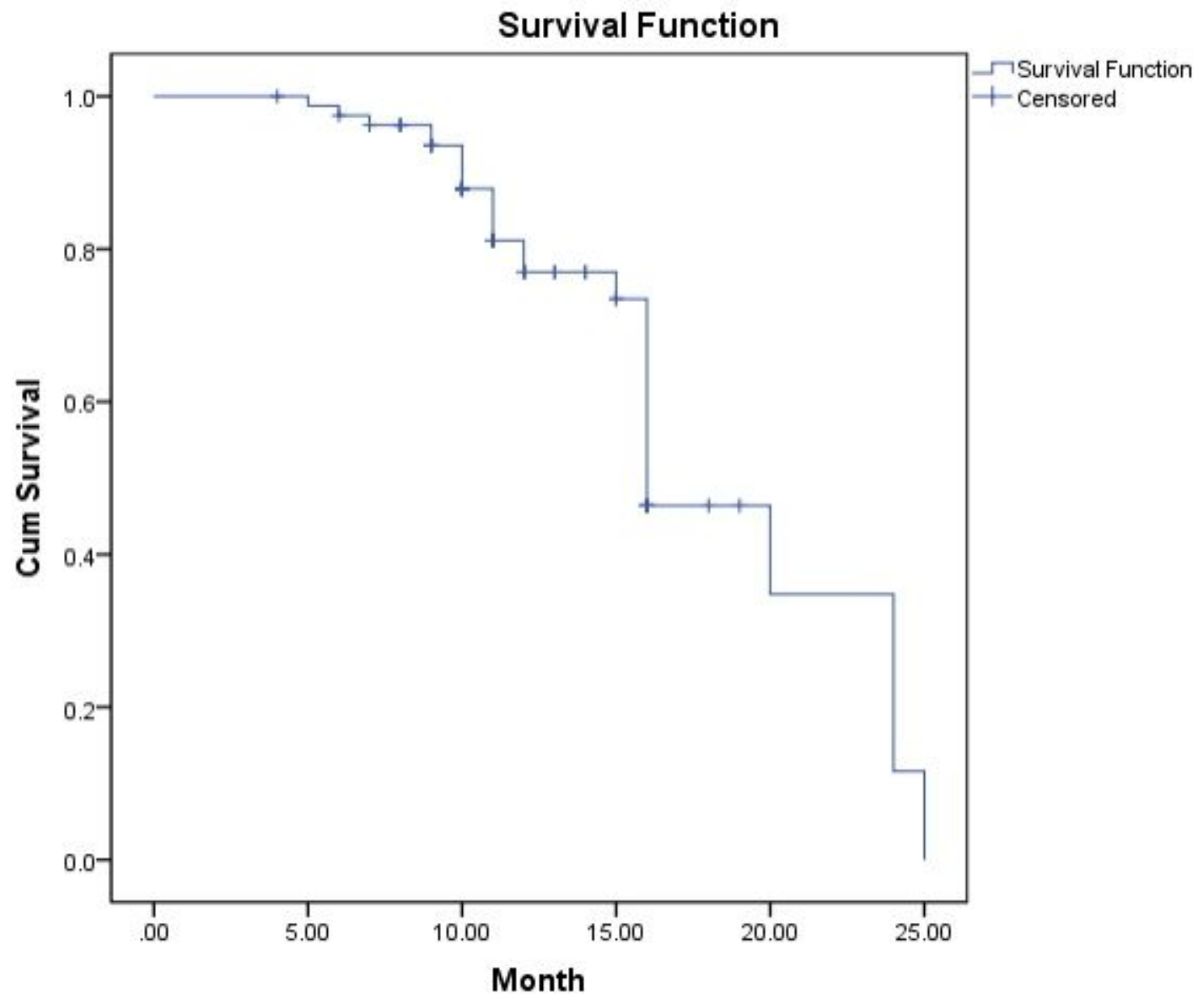

Figure 4

Median postoperative survival of patients

\section{Supplementary Files}

This is a list of supplementary files associated with this preprint. Click to download.

- table2.xls

- table1.xls

- table3.xls 